Conclusion Rheumatic diseases are a very prevalent problem in the primary health care level in Portugal and have an important repercussion in the social and economic field.

\section{AB0226 PREVALENCE OF RADIOGRAPHIC HIP OSTEOARTHRITIS IN TURKEY}

B Goker. Internal Medicine, Gazi University Medical School, Ankara, Turkey

\subsection{6/annrheumdis-2001.774}

Background The prevalence of hip osteoarthritis (OA) varies greatly across different geographic locations and population groups. Limited data exist about the prevalence of radiographic hip OA in Turkey.

Objectives The aim of the study was to assess the prevalence of radiographic hip OA in Turkish population.

Methods The radiographs kept at the files of the Department of Urology at Gazi University/Ankara were accessed. Patients aged 25 and over were included (range:25-97). Plain supine abdominal radiographs and intravenous pyelographies were evaluated. A hip joint was included in the study only if the entire femoral head and acetabulum were visible. Kellgren and Lawrence (K\&L) grading system for OA was utilised.

Results 1249 hips in 682 (205F, 477M) patients were evaluated. Overall, $8.8 \%$ of the hips evaluated had radiographic hip OA (K\&L grade 2 and above). In 566 patients, both hips were included, and of these, $11.7 \%$ had radiographic hip OA in either one or both hips (9.4\% of women and $12.7 \%$ of men). $51.5 \%$ of the cases had bilateral findings. The prevalence of radiographic hip OA increased with age. It was $1.9 \%, 16.1 \%$, and $21.6 \%$ for age ranges $25-39,40-54$ and $>54$, respectively. In the majority of the patients the radiographic findings indicated only mild disease (K\&L grade 2 ). There were 3 patients with grade 3 (moderate) disease and only 1 patient (aged 68) had K\&L grade 4 (severe) OA.

Conclusion The results suggest that, K\&L grade 2 OA changes emphasising osteophytes at the hip joints are not uncommon in Turkish population, especially among aged 40 and over, but moderate-to-severe radiographic $\mathrm{OA}$ is not frequent.

\section{AB0227 SCREENING AND DIAGNOSTIC TESTS OF TYROIDAL DISFUNCTION: ACTIVITY OF A RHEUMATOLOGY UNIT IN A YEAR OF FOLLOW-UP}

${ }^{1} \mathrm{MM}$ Martinez-Losa, ${ }^{1} \mathrm{HC}$ Macias, ${ }^{2} \mathrm{E}$ Urgell, ${ }^{2} \mathrm{JR}$ Espinosa, ${ }^{1} \mathrm{CD}$ Lopez, ${ }^{1} \mathrm{CG}$ Ferrer, ${ }^{1} \mathrm{JM}$ Llobet, ${ }^{1}$ AR De la Serna. ${ }^{1}$ Rheumatology; ${ }^{2}$ Biochemistry, Hospital de La Santa Creu I Sant Pau, Barcelona, Spain

\subsection{6/annrheumdis-2001.775}

Background Tyroidal disfunction has been widely related with autoimmune diseases and its prevalence is higher $(23 \%)$ than the one described in normal population (10\%). Tyroidal function tests are a common procedure in our rheumatological daily activity but protocols must be performed to detect cases at early onset.

Objectives Screening tyroid tests and its diagnostic value in a Rheumatology Unit.

Methods Between January and December (1998) 10.992 outpatients were visited in our Rheumatology Unit (range of age:1891). Among them 602 tyroid function tests were performed
(5.5\%). Treatment with drugs that could interfere tyroidal function were also considered.

Results A higher frequency of tyroid disfunction was observerd in hospitalised patients (31\%) than in outpatients $(13 \%)(\mathrm{p}<$ $0.05)$. The majority of new cases $(14 \%$; IC $95: 11-17 \%)$ were subclinical $(12.5 \%$;IC95:10-16\%) and no significant differences were seen between groups of age (59) and sex. New cases described according to diagnostic: see Table 1 (RA: Rheumatoid Arthritis, SS: Sjögre Syndrom, Arth: Arthritis, Scl: Scleroderma, LES: Systemic Lupus Erythematosus, FM; Fybromialgia, OA: Osteoarthritis, Artg: Arthralgia, Mig: Mialgia, T. Disf: Tyroidal disfunction).

\begin{tabular}{lllllllll}
\multicolumn{6}{c}{ Abstract AB0227 Table 1 } \\
\hline \multicolumn{2}{c}{ RA } & SS & Arth & $\begin{array}{l}\text { Scl } \\
\text { +LES }\end{array}$ & FM & OA & Artg & Mig \\
\hline T. Disf & $12 / 70$ & $22 / 138$ & $3 / 65$ & $8 / 29$ & $6 / 47$ & $8 / 23$ & $3 / 24$ & $2 / 30$ \\
$(\mathbf{n} \%)$ & $(17 \%)$ & $(16 \%)$ & $(4.6 \%)$ & $(27.6 \%)$ & $(12.7 \%)$ & $(35 \%)$ & $(12.5 \%)$ & $(6.7 \%)$ \\
$<60$ & $5 / 32$ & $11 / 59$ & $3 / 38$ & $6 / 21$ & $5 / 35$ & $0 / 2$ & $3 / 14$ & $0 / 17$ \\
& $(15.6 \%)$ & $(18.6 \%)$ & $(7.9 \%)$ & $(28.5 \%)$ & $(14.3 \%)$ & $(0.0 \%)$ & $(21.4 \%)$ & $(0.0 \%)$ \\
$>59$ & $7 / 28$ & $11 / 79$ & $0 / 27$ & $2 / 8$ & $1 / 12$ & $7 / 21$ & $0 / 10$ & $2 / 13$ \\
& $(18.4 \%)$ & $(14 \%)$ & $(0.0 \%)$ & $(25 \%)$ & $(8.3 \%)$ & $(33.3 \%)$ & $(0.0 \%)$ & $(15.4 \%)$ \\
\hline
\end{tabular}

Conclusion These results confirm that the study for tyroidal autoimmunity should be performed to clarify diagnosis, treatment and prognosis of this group of rheumatic patients due to its higher prevalence than in general population.

\section{AB0228 PERIPHERAL FRACTURES AND BONE DENSITY OF THE LUMBAR SPINE IN WOMEN}

NV Toroptsova, NV Demin, LI Benevolenskaya. Epidemiology and Genetics, Institute of Rheumatology, Moscow, Russia

\subsection{6/annrheumdis-2001.776}

\section{Background}

Objectives The purpose of the study was to determine the prevalence of osteoporosis in lumbar spine and peripheral fractures after 40 years in women 55 and elder.

Methods Cross-sectional study of 2155 women using bone mineral density (BMD) with DEXA (Hologic 4500 A and W). Retrospective fracture history after 40 years was collected by interview.

Results The prevalence of osteoporosis in lumbar spine according WHO-criteria was $29,8 \%$. Fractures due fall were found in $31 \%$ of women. Over $50 \%$ of the fractures were of the wrist. There were no significant differences in age, anthropometric properties, number of children, age of menopause and duration of menopause between patients with retrospective fracture (RF) history and without fractures (WF). The prevalence of osteoporosis in lumbar spine in RF vs WF was 32\% and 17\% respectively. There was significant difference in the BMD of lumbar spine between RF and WF groups $\left(0,828 \mathrm{~g} / \mathrm{cm}^{2}\right.$ and $0,874 \mathrm{~g} / \mathrm{cm}^{2}$ respectively, $\mathrm{p}<0,001$ ).

Conclusion Our study presents the high prevalence of osteoporosis in lumbar spine in postmenopausal women. We found negative association between BMD of lumbar spine and peripheral fractures, so the patients with peripheral fractures are at high risk of osteoporosis, who needs BMD measurements. 